\title{
Endoscopic Removal of Chondrosarcoma Originating from the Nasal Septum
}

\author{
Tatsunori Sakamoto*, Takayuki Nakagawa and Juichi Ito \\ Department of Otolaryngology, Head and Neck Surgery, Graduate School of Medicine, Kyoto University, Kawaharacho \\ 54, Shogoin, Sakyo-ku, Kyoto, 606-8507, Japan
}

\begin{abstract}
Background: Chondrosarcomas are rare non-epithelial malignant tumours. Surgery is the primary treatment option for chondrosarcoma, with adjuvant therapy having a minor role. In cases of nasal chondrosarcoma, however, en bloc total removal by open approaches is often undesirable. Here we report a case of chondrosarcoma arising from the nasal septum, which was resected endoscopically.

Case Report: The case was 50-year-old man with nasal stuffiness. Computed tomography revealed a large lesion arising from the nasal septum composed of a low-density area with scattered high-density areas. Grade I chondrosarcoma was diagnosed based on histopathological analysis. After tumour margins were carefully identified on CT images and in the nasal cavities, trans-nasal endoscopic piece-by-piece tumour removal was conducted. One year after the surgery, there was no sign of local recurrence or distant metastasis.

Clinical Implication: Transnasal endoscopic removal could represent a safe and potentially curative treatment for this malignant tumour.
\end{abstract}

Keywords: Case report, chondrosarcoma, endoscopic nasal surgery, nasal septum.

\section{INTRODUCTION}

Chondrosarcomas are rare slow-growing non-epithelial malignant tumours that usually arise from cartilaginous structures. Only about 50 cases of nasal septum chondrosarcomas are reported in the English literature [1]. Surgery is the primary treatment option for chondrosarcoma. In cases of nasal chondrosarcoma, however, en bloc total removal by open approaches is often undesirable due to the considerable risk of morbidity and unavoidable cosmetic and functional problems. Endoscopic removal should be favourable for avoiding external excisions and limiting the morbidity. Here we report a case of chondrosarcoma of the nasal septum that was successfully treated by transnasal endoscopic surgery.

\section{CASE REPORT}

A 50-year-old man complaining of nasal stuffiness was followed up conservatively by a local otorhinolaryngologist as a case with chronic rhinosinusitis for several years. Stuffiness was the only symptom for him. He was referred to our department for further evaluation and treatment of a huge mass in his nasal cavity, which had been revealed unexpectedly by screening computed tomography (CT) after head trauma. A large bony hard mass was found in his nasal cavities. Axial, coronal and sagittal CT images revealed a large lesion arising from the posterior-inferior part of the nasal septum (Fig. 1a-c) and to be composed of a low-

*Address correspondence to this author at the Department of Otolaryngology, Head and Neck Surgery, Graduate School of Medicine, Kyoto University, Kawaharacho 54, Shogoin, Sakyo-ku, Kyoto, 606-8507, Japan; Tel: +81/75-751-3346; Fax: +81/75-751-7225;

E-mail: sakamoto@ent.kuhp.kyoto-u.ac.jp density (soft-tissue) area with scattered high-density (bony) areas. The left nasal floor (that is, the left palatal bone) and the anterior wall of the sphenoid sinuses were involved; however, the cribriform plate, sella turcica, clivus, and medial walls of the left and right orbits were tumour free. Although the right lateral wall of the nasal cavity was displaced laterally, its involvement was unclear, because the bony structure of the middle turbinate remained identifiable. T1-weighted and T2-weighted magnetic resonance (MR) images revealed that the lesion contained areas of different densities (Fig. 1d, T1 images are not shown). Based on histopathological analysis of specimens from the right nasal cavity, grade I chondrosarcoma was diagnosed.

Tumour resection was conducted by nasal endoscopy under general anaesthesia. The tumour margins were identified at the beginning of the surgery. There was a narrow gap between the tumour and the nasal floor in the right nasal cavity. The tumour was continuous with the nasal floor in the left nasal cavity. The left sphenoid sinus was inspected through its enlarged natural ostium and the posterior wall was confirmed to be tumour free. The tumour continuous with the left nasal floor was drilled out without perforating the oral cavity. The tumor was totally removed piece-by-piece using microdebriders, burrs and forceps. The right middle and inferior turbinates, the medial wall of the right and left orbits, the left middle and inferior turbinates, and mucosa of cribriform plate and fovea ethmoidalis were tumour free. There was no need for the ligation of sphenopalatine artery. Volume of intraoperative hemorrhage was $245 \mathrm{ml}$, and the procedure took 3 hours and 45 minutes. The removed specimens were composed of cartilageous and fibrous tissue which is compatible to chondrosarcoma. (Fig. 2) No additional adjuvant treatment was added. 

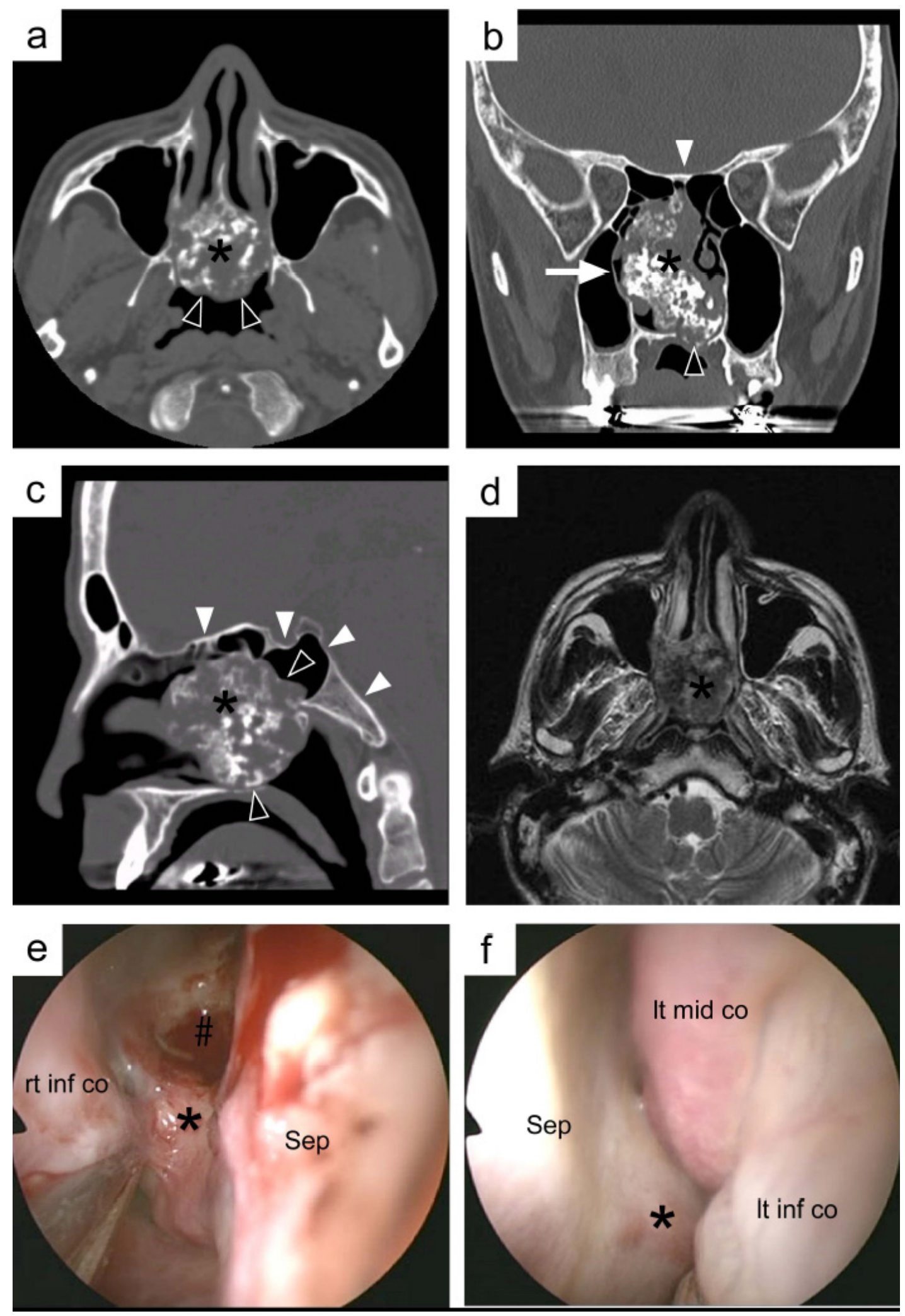

Fig. (1). Preoperative CT, MR and endscopic images. Axial (a), coronal (b) and sagittal (c) views of CT images revealed a large lowdensity area with scattered bony high-density spots originating from the nasal septum $(*)$. Note that the left palatal bone (black arrowheads in $\mathrm{b}$ and $\mathbf{c}$ ) and the anterior wall of the sphenoid sinuses (black arrowhead in a) were involved, whereas the cribriform plate, the sella turcica, the posterior wall of the sphenoid sinus and the clivus (white arrowheads in $\mathbf{c}$ ), and the medial walls of the left and right orbits were not involved. The lateral wall of the right nasal cavity (white arrowhead in $\mathbf{b}$ ) was displaced laterally. The lesion $(*)$ showed a moderate-to-high intensity signal on T2-weighted MR images (d). Endoscopic views of right (e) and left (f) nasal cavities at the beginning of the surgery. The lesion $(*)$ was continuous to the nasal sepatum (Sep) on both sides, but was free from the right inferior concha ( $\mathrm{rt}$ inf co), right nasal floor, left middle and inferior conchae (lt mid/inf co). A part of the lesion (\#) was removed at the time of biopsy. 


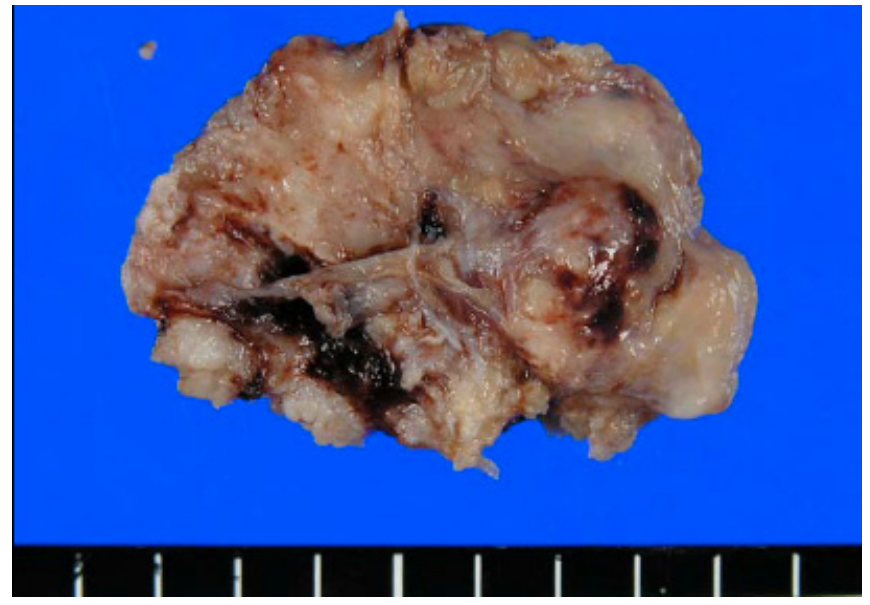

Fig (2). Macroscopic view of a large piece of the tumor. The tumor was composed of cartilageous and fibrous tissue. Scale markings were shown every $5 \mathrm{~mm}$.

Three months after the surgery, no metastatic hot spot could be detected on positron emission tomography (PET) images. One year and 7 months after the surgery, no tumour could be detected in the nasal cavity by nasal endoscopy (Fig 3a) and on CT images (Fig 3b, c).

\section{DISCUSSION}

Chondrosarcomas are rare slow-growing non-epithelial malignant tumours. They are most prevalent in the pelvic bone, femur and humerus $[2,3]$, and only $7.4 \%$ develop in the nasal region (including the nasal cavity, septum and sinuses) [2]. Surgery is the primary option for the treatment of chondrosarcomas, with adjuvant therapy having a minor role [1]. Uncontrollable local diseases are the most common cause of death in these cases [4], and the rate of local recurrence is primarily dependent on the adequacy of surgical removal [2]. These findings point towards open approaches for complete removal. However, in cases of nasal chondrosarcoma, extensive en bloc removal by open approaches, such as lateral rhinotomy, anterior craniofacial resection with or without facial dismasking and Le Fort I down-fracture [5-7], is often undesirable because of functional and cosmetic problems and significant associated morbidity. We chose the transnasal endoscopic piece-bypiece removal of the tumour in this case, on the basis of the superior cosmetic and functional results, the low rate of distant metastasis, and the resectable tumour margins. The histological grading by cellularity, nuclear size and mitotic rate is reported to correlate with the rate of distant metastasis and overall survival [2]. Grade I chondrosarcoma, like the
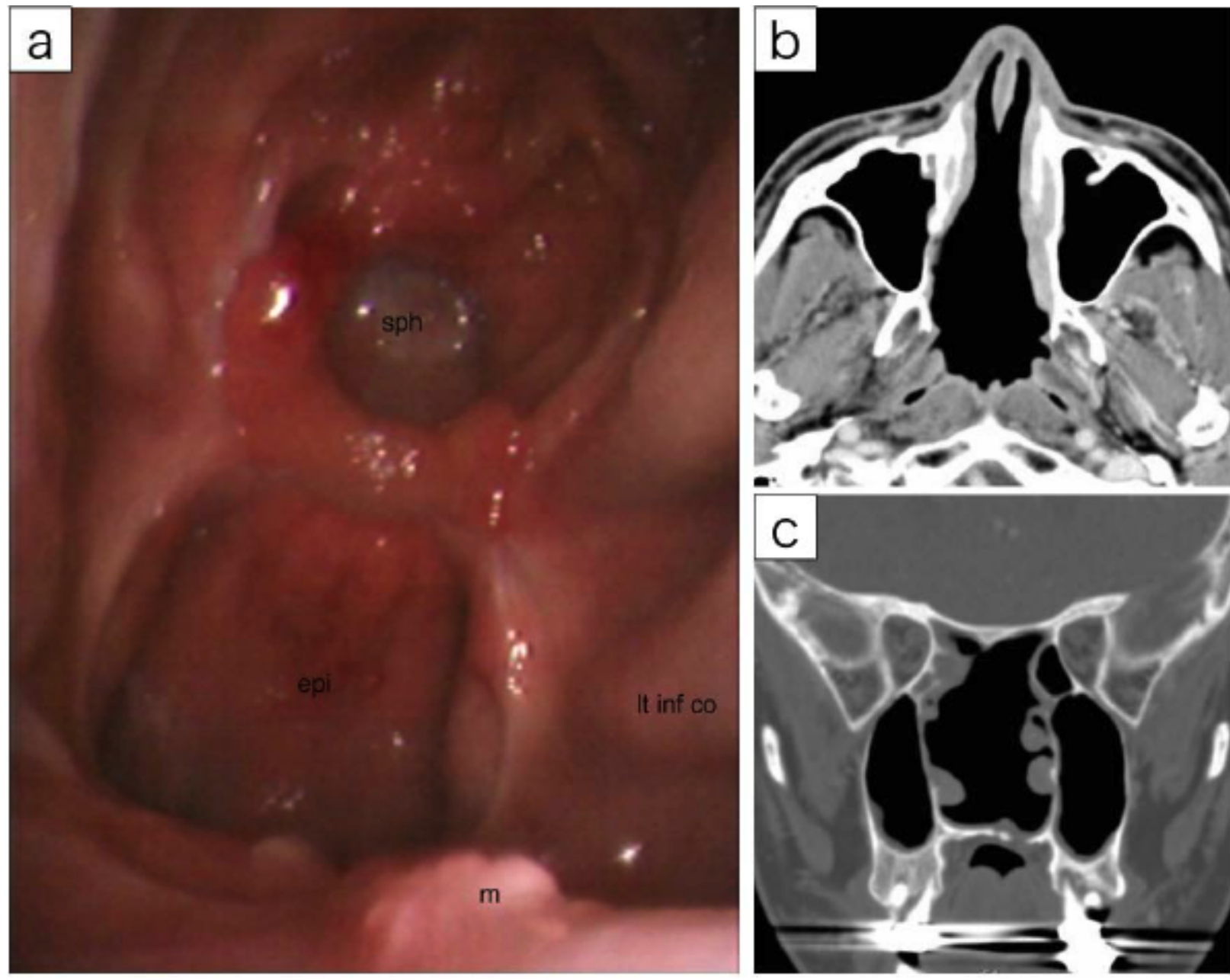

Fig. (3). Postoperative fiberscopic view and CT images. There was no residual or recurrent tumour on endoscopic view (a), and axial (b) and coronal (c) CT images 12 months after surgery. : sphenoid sinus (sph), epipharynx (epi), maxillary crest (m), and left inferior nasal concha (lt inf co). 
tumour in the present case, is associated with a low probability of distant metastasis and good survival. The CT and MR images were carefully inspected to determine the surgical margins. We predicted that the tumour could be completely removed using nasal endoscopy, because the skull base was tumour free, although the left nasal floor and the anterior walls of the sphenoid sinuses were involved. The margins were confirmed in the early phase of the surgery. Good local control was achieved for at least 1 year postoperatively, however, this slow glowing tumour may recur after several years. It would be necessary to continue to follow-up.

The English literature includes three reports describing four cases of endoscopic removal of nasal septum chondrosarcomas, in all of which clear tumour margins were confirmed by preoperative CT scans and good local control was achieved $[1,6,8]$. At present, we consider cases without skull-base involvement to be good indications for transnasal endoscopic removal. More aggressive transnasal skull-base surgeries have been reported in the literature [9-12]. We believe that transnasal endoscopic removal for this type of tumour should be included as a primary option in the future, even for more extensive cases.

\section{CONCLUSION}

A rare case of nasal septum chondrosarcoma was successfully treated by endoscopic nasal surgery. This approach could represent a safe and potentially curative treatment for this malignant tumour.

\section{ACKNOWLEDGEMENTS}

We would like to thank Dr. Yasuaki Nakashima from the Laboratory of Anatomic Pathology, Kyoto University
Hospital, Japan, for the histopathological diagnosis, and Dr. Takeharu Nakamata and Dr. Hiroyuki Yoshitomi from the Department of Orthopaedic surgery, Kyoto University Hospital, Japan, for constructive discussions.

\section{REFERENCES}

[1] Coppit GL, Eusterman VD, Bartels J, Downey TJ. Endoscopic resection of chondrosarcomas of the nasal septum: a report of 2 cases. Otolaryngology 2002; 127: 569-71.

[2] Evans HL, Ayala AG, Romsdahl MM. Prognostic factors in chondrosarcoma of bone: a clinicopathologic analysis with emphasis on histologic grading. Cancer 1977; 40: 818-31.

[3] Ollivier L, Vanel D, Leclere J. Imaging of chondrosarcomas. Cancer Imaging 2004; 4: 36-8.

[4] Ruark DS, Schlehaider UK, Shah JP. Chondrosarcomas of the head and neck. World J Surg 1992; 16: 1010-6.

[5] Downey TJ, Clark SK, Moore DW. Chondrosarcoma of the nasal septum. Otolaryngology 2001; 125: 98-100.

[6] Kaufman JK, Pritz MB, Righi PD, Bizal JC. Craniofacial resection of a nasoseptal chondrosarcoma: case report and review of the literature. Surg Neurol 1999; 52: 265-9.

[7] Nishimura Y, Amano Y, Ogasawara H. Chondrosarcoma of the nasal septum: surgical considerations on Le Fort I osteotomy. Eur Arch Otorhinolaryngol 1993; 250: 59-62.

[8] Matthews B, Whang C, Smith S. Endoscopic resection of a nasal septal chondrosarcoma: first report of a case. Ear Nose Throat J 2002; 81: 327-9.

[9] Stamm AC, Pignatari S, Vellutini E, Harvey RJ, Nogueira JF. A novel approach allowing binostril work to the sphenoid sinus. Otolaryngology 2008; 138: 531-2.

[10] Stamm AC, Pignatari SS, Vellutini E. Transnasal endoscopic surgical approaches to the clivus. Otolaryngol Clin North Am 2006; 39: 639-56.

[11] Stamm AC, Vellutini E, Harvey RJ, Nogeira JF, Herman DR Endoscopic transnasal craniotomy and the resection of craniopharyngioma. Laryngoscope 2008; 118: 1142-8.

[12] Stamm AM. Transnasal endoscopy-assisted skull base surgery. Ann Otol Rhinol Laryngol Suppl 2006; 196: 45-53. 